\title{
Size effects of ferroelectric and magnetoelectric properties of semi-ellipsoidal bismuth ferrite nanoparticles
}

\author{
Victoria V. Khist ${ }^{1}$, Eugene A. Eliseev ${ }^{2}$, Maya D. Glinchuk ${ }^{2}$, Maxim V. Silibin ${ }^{3}$, Dmitry V. \\ Karpinsky ${ }^{4 *}$, and Anna N. Morozovska ${ }^{5 \%}$ \\ ${ }^{1}$ Institute of Magnetism, National Academy of Sciences of Ukraine and Ministry of Education and \\ Science of Ukraine, \\ ${ }^{2}$ Institute for Problems of Materials Science, National Academy of Sciences of Ukraine, \\ 3, Krjijanovskogo, 03142 Kyiv, Ukraine, \\ ${ }^{3}$ National Research University of Electronic Technology "MIET”, \\ Moscow, Zelenograd, Russia \\ ${ }^{4}$ Scientific-Practical Materials Research Centre of NAS of Belarus, Minsk, Belarus \\ ${ }^{5}$ Institute of Physics, National Academy of Sciences of Ukraine, \\ 46, pr. Nauky, 03028 Kyiv, Ukraine
}

\begin{abstract}
Bismuth ferrite $\left(\mathrm{BiFeO}_{3}\right)$ is one of the most promising multiferroics with a sufficiently high ferroelectric (FE) and antiferromagnetic (AFM) transition temperatures, and magnetoelectric (ME) coupling coefficient at room temperature. So $\mathrm{BiFeO}_{3}$ is highly sensitive to the impact of cross-influence of applied electric and magnetic fields. According to the urgent demands of nanotechnology to miniaturize the working elements for ultra-high density data storage in advanced nonvolatile memory cells, it is very important to reduce the sizes of multiferroic nanoparticles in the self-assembled arrays without serious deterioration of their properties. This work studies size effects of the phase diagrams, $\mathrm{FE}$ and ME properties of semi-ellipsoidal $\mathrm{BiFeO}_{3}$ nanoparticles clamped to a rigid conductive substrate. The spatial distribution of the spontaneous polarization vector inside the nanoparticles, phase diagrams and paramagnetoelectric (PME) coefficient were calculated in the framework of the modified Landau-Ginzburg-Devonshire (LGD) approach. Analytical expressions were derived for the dependences of the FE transition temperature, average polarization, linear dielectric susceptibility and PME coefficient on the particle sizes for a general case of a semi-ellipsoidal nanoparticles with different semi-axes $a, b$ and height $c$. The analyses of the obtained results leads to the conclusion that the size effect of the phase diagrams, spontaneous polarization and PME coefficient is rather sensitive to the aspect ratio of particle sizes in the polarization direction, and less sensitive to the absolute values of the sizes.
\end{abstract}

\footnotetext{
* corresponding author, e-mail: dmitry.karpinsky@gmail.com

${ }^{\dagger}$ corresponding author, e-mail: anna.n.morozovska@gmail.com
} 
Keywords: Multiferroics; Structural antiferrodistortion; Antiferromagnetic order; Nanoparticle; Semiellipsoidal; Nano-islands.

\section{Introduction}

\subsection{Multiferroic $\mathrm{BiFeO}_{3}$ for fundamental studies and advanced applications}

Multiferroics, which are ferroics with two or more interacting long-range order parameters, are ideal systems for fundamental studies of the couplings among the ferroelectric (FE) polarization, structural antiferrodistortive (AFD), and antiferromagnetic (AFM) order parameters [1-5]. These couplings are in response of unique physical properties of multiferroics [6]. For instance, biquadratic and linear magnetoelectric (ME) couplings lead to intriguing effects, such as a giant ME tunability of multiferroics [7]. Biquadratic coupling of the structural, polar and dielectric order parameters introduced in Refs. [8,9], are responsible for the unusual behavior of the dielectric, polar and other physical properties in ferroelastics-quantum paraelectrics. The linear-quadratic paramagnetoelectric (PME) effect should exist in the paramagnetic phase of all ferroics, below the temperature of the paraelectric to ferroelectric phase transition, where the electric polarization is nonzero. This effect was observed in $\mathrm{NiSO}_{4} \cdot 6 \mathrm{H}_{2} \mathrm{O}$ [10], Mn-doped $\mathrm{SrTiO}_{3}$ [11], $\mathrm{Pb}\left(\mathrm{Fe}_{1 / 2} \mathrm{Nb}_{1 / 2}\right) \mathrm{O}_{3}$ [12], and $\mathrm{Pb}\left(\mathrm{Fe}_{1 / 2} \mathrm{Nb}_{1 / 2}\right) \mathrm{O}_{3}-\mathrm{PbTiO}_{3}$ solid solution [13]. Note that PME effect can be expected in many nanosized ferroics, which becomes paramagnetic due to the size-induced transition from the ferromagnetic or antiferromagnetic phase.

$\mathrm{BiFeO}_{3}$ is the one of the most interesting multiferroics with a strong ferroelectric polarization, antiferromagnetism at room temperature [4, 5], local ferromagnetism [14], spin-lattice coupling phase transition and phonon anomalies [15] as well as enhanced electrotransport properties at domain walls [16-21]. Bulk $\mathrm{BiFeO}_{3}$ exhibits AFD order at temperatures below 1200K; it is FE order with a large spontaneous polarization below $1100 \mathrm{~K}$ and AFM with a cycloid spin order below Neel temperature $T_{N} \approx 650 \mathrm{~K}[22,23]$. The pronounced multiferroic properties maintain in $\mathrm{BiFeO}_{3}$ thin films [24-27] and heterostructures [28]. Despite extensive experimental and theoretical studies of the physical properties of bulk $\mathrm{BiFeO}_{3}$ and its thin films [20-22, 29-35] many important issues concerning the emergence and theoretical background of multiferroic, polar, magnetic and various electrophysical properties of $\mathrm{BiFeO}_{3}$ nanoparticles remain almost unexplored $[36,37]$.

\subsection{Multiferroelectric nanoparticles. State-of-art}

According to the urgent demands of nanotechnology to miniaturize the working elements for ultra-high density of data storage in advanced nonvolatile memory cells, it is very important to reduce the sizes of multiferroic nanoparticles in the self-assembled arrays without serious 
deterioration of their FE, AFM and ME properties. There are many intriguing and encouraging examples of the FE and ME properties conservation, enhancement and modification in ferroelectric nanoparticles. In particular Yadlovker and Berger [38] presented the unexpected experimental results, which reveal the enhancement of FE properties of cylindrical nanoparticles of Rochelle salt. Zhao et al [39] and Erdem et al [40] demonstrated the possibility to control the temperature of the ferroelectric phase transition, the magnitude and position of the dielectric constant maximum for $\mathrm{BaTiO}_{3}$ and $\mathrm{PbTiO}_{3}$ nanopowders and nanoceramics. The studies of $\mathrm{KTa}_{1-\mathrm{x}} \mathrm{Nb}_{\mathrm{x}} \mathrm{O}_{3}$ nanopowders and nanograin ceramics [41] discover the appearance of novel FE phases, the shift of FE transition temperature in comparison with bulk crystals. Strong size effects in $\mathrm{SrBi}_{2} \mathrm{Ta}_{2} \mathrm{O}_{9}$ nanoparticles have been revealed by in situ Raman scattering by Yu et al [42] as well as by thermal analysis and Raman spectroscopy by Ke et al [43].

Size-dependent ferroelectric phase transition, magnetic properties [44], infrared phonon modes [45], optical and photocatalytic properties [46] have been observed in $\mathrm{BiFeO}_{3}$ nanoparticles. $\mathrm{BiFeO}_{3}$ nano-island can be promising candidates for resistive switching memory [47]. The physical properties of nanosized $\mathrm{BiFeO}_{3}$ can be controlled and tuned by doping with different rare-earth metals. For instance, the co-substitution of $\mathrm{Gd}$ and $\mathrm{Mn}$ in $\mathrm{BiFeO}_{3}$ nanoparticles leads to the magnetization saturation and band gap tuning [48]. $\mathrm{Nd}$ and $\mathrm{Nb}$ co-doping of $\mathrm{BiFeO}_{3}$ nanoparticles strongly affects on their structural, magnetic and optical properties [49]. Fe doped $\mathrm{BaZrO}_{3}$ nanoceramic is a room temperature ferromagnetic [50]. The list of experimental studies should be continued making any new experimental and theoretical study of ferroelectric nanoparticles important for both fundamental science and applications.

In particular, the surface and finite size effects impact on the phase diagrams, polar and electrophysical properties of $\mathrm{BiFeO}_{3}$ nanoparticles are very poorly studied $[36,37]$. Such study may be very useful for science and applications, because the theory of finite size effects in nanoparticles allows one to establish the physical origin of the properties anomalies, transition temperature and phase diagrams changes appeared with the sizes decrease. In particular, using the continual phenomenological approach Niepce [51], Huang et al [52, 53], Ma [54], Eliseev et al [55] and Morozovska et al [56-60] have shown, that the changes of the transition temperatures, the enhancement or weakening of FE properties in spherical and cylindrical nanoparticles are conditioned by the various physical mechanisms, such as correlation effect, depolarization field, flexoelectricity, electrostriction, surface tension and Vegard-type chemical pressure.

\subsection{Research motivation}

Nanoparticles of semi-ellipsoidal shape can be considered as the model objects to study the size effects of physical properties in ferroic nano-islands. $\mathrm{BiFeO}_{3}$ nano-islands and their selfassembled arrays can be formed on anisotropic substrates by different low-damage fabrication 
methods [61-63]. The particles typically have different in-plane axes due to the anisotropic thermal conductivity of substrate. Recent advances in the production technology of ferroelectrics have resulted in a cost-effective synthesis of these nanoparticles, which are beginning to be used in fabrication of microactuators, microwave phase shifters, infrared sensors, transistor applications, energy harvesting devices, etc. A correlation mechanism between the scaling factor, geometry of the nanoparticles and their physical parameters, and related phenomena viz. spontaneous FE polarization, AFM and AFD order, width of the domain walls and the domains stability is needed to be further investigated using both experimental methods and theoretical modeling. Most intriguing fundamental issues to be addressed include the estimation of the intrinsic limit for FE polarization stability, mechanism of domain wall motion, and polarization reversal in nanoscale regions [1-6].

The analyses of the above state-of-art motivated us to study theoretically the size effects influence on FE, $\mathrm{AFM}$ and $\mathrm{ME}$ properties of semi-ellipsoidal $\mathrm{BiFeO}_{3}$ nanoparticles in the framework of the Landau-Ginzburg-Devonshire (LGD) approach, classical electrostatics and elasticity theory.

\section{Theory}

\subsection{Problem statement}

It is known that ferroelectricity is a cooperative phenomenon associated with the dipole moments aligned on both short-range and long-range scale level. This alignment is characterized by a certain transition temperature justified by the temperature-dependent forces, which are related with the size effects, dimension of the material, its structural homogeneity etc. It is accepted that the size effects are associated either with intrinsic (mainly related to the atomic polarization) or extrinsic (stresses, microstructure, polarization screening etc.) factors [56-59].

Let us consider ferroelectric nanoparticles in the form of semi-elliptical islands precipitated on the rigid conducting substrate electrode. The ellipsoid has different values of semi-axis length, $a$, $b$ and height $c$ along $\mathrm{X}$-, $\mathrm{Y}$ - and Z-axis, respectively. We denote $\varepsilon_{b}$ and $\varepsilon_{e}$ as the dielectric permittivity of ferroelectric background and external media, respectively. The one-component ferroelectric polarization $\mathbf{P}(\mathbf{r})$, directed along the crystallographic axis 3 inside the particle, that is parallel to the interface $\mathrm{z}=0$ [Fig. 1]. 


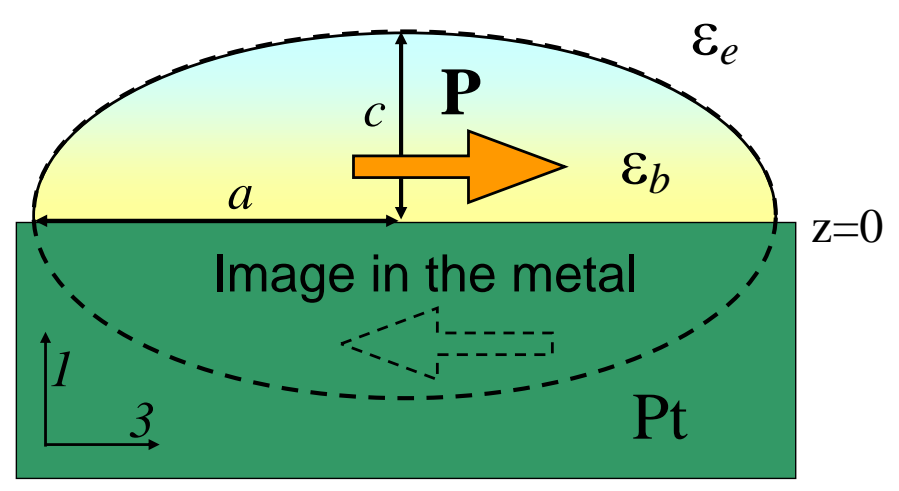

Fig. 1. A semi-ellipsoidal uniformly polarized ferroelectric nanoparticle is clamped to a rigid conducting substrate electrode (e.g., Pt). The one-component ferroelectric polarization $\mathbf{P}(\mathbf{r})$ is directed along x-axis. Semi-ellipsoid height is $c$ and lateral semi-axes are $a$ and $b$. The considered crystal system is pseudo-cubic.

The considered crystal system is pseudo-cubic with the crystallographic axes $\{1,2,3\}$. Since we are working in the LGD approach, in which framework the equations of state are derived from the variation of free energy functional, the functional is a continuous function of polarization in the pseudo-cubic parent phase without long-range FE and AFM orders as well as in the longrange ordered rhombohedral ferroelectric phase. Therefore true symmetry of the free energy functional corresponds to the irreducible representation of the pseudo-cubic parent phase symmetry. The symmetry is broken spontaneously with the appearance of the order parameter with the temperature decrease.

Hence we can assume that in the pseudo-cubic crystallographic frame $\{1,2,3\}$ the dependence of the in-plane components of electric polarization on the inner field electric $\mathbf{E}^{i}$ is linear $P_{1}=\varepsilon_{0}\left(\varepsilon_{b}^{i}-1\right) E_{1}^{i}$ and $P_{2}=\varepsilon_{0}\left(\varepsilon_{b}^{i}-1\right) E_{2}^{i}$, where an isotropic background permittivity is relatively small, $\varepsilon_{b}^{i} \leq 10$ [1], $\varepsilon_{0}$ is a universal dielectric constant. Polarization component 3 contains background and soft mode contributions, $P_{3}\left(\mathbf{r}, E_{3}\right)=P\left(\mathbf{r}, E_{3}\right)+\varepsilon_{0}\left(\varepsilon_{b}^{i}-1\right) E_{3}^{i}$. Electric displacement vector has the form $\mathbf{D}^{i}=\varepsilon_{0} \varepsilon_{b}^{i} \mathbf{E}^{i}+\mathbf{P}$ inside the particle and $\mathbf{D}^{e}=\varepsilon_{0} \varepsilon^{e} \mathbf{E}^{e}$ outside it; $\varepsilon^{e}$ is the relative dielectric permittivity of external media. Hereinafter the subscript " $i$ " corresponds to the electric field or potential inside the particle, " $e$ " corresponds to the values outside the particle.

Inhomogeneous spatial distribution of the $\mathrm{FE}$ polarization component $P_{3}\left(\mathbf{r}, E_{3}\right)$ can be determined from the LGD-type equation inside a nanoparticle,

$$
\alpha_{P} P_{3}+\beta_{P} P_{3}^{3}+\gamma_{P} P_{3}^{5}-g_{33 m n} \frac{\partial^{2} P_{3}}{\partial x_{m} \partial x_{n}}-2 Q_{k l i 3} \sigma_{k l} P_{3}=E_{3},
$$

where the coefficient $\alpha_{P}(T)=\alpha_{P}^{(T)}\left(T-T_{C}\right), T$ is the absolute temperature, $T_{C}$ is the Curie temperature of the FE phase transition. The parameters $\beta_{P}$ and $\gamma_{P}$ are coefficients of LGD potential 
expansion on the polarization powers. $\sigma_{k l}$, and $Q_{i j k l}$ are respectively elastic stress and electrostriction stress tensor. Flexoelectric effect is regarded as small. Boundary conditions for the polarization $P_{3}$ at the particle surface $\mathrm{S}$ are regarded to be natural, $\left.\left(\partial P_{3} / \partial \mathbf{n}\right)\right|_{S}=0$.

Electric field $E_{i}$ is defined via electric potential as $E_{i}=-\partial \varphi / \partial x_{i}$. For a ferroelectric nanoparticle without free charge carriers, the electric potential $\varphi$ can be found self-consistently from the Laplace equation outside the nanoparticle $\varepsilon_{0} \varepsilon^{e} \Delta \varphi_{e}=0$ and Poisson equation inside it

$$
\varepsilon_{0} \varepsilon_{i j}^{b} \frac{\partial^{2} \varphi}{\partial x_{i} \partial x_{j}}=\frac{\partial P_{k}}{\partial x_{k}}
$$

$\varepsilon_{0}=8.85 \times 10^{-12} \mathrm{~F} / \mathrm{m}$ the dielectric permittivity of vacuum, $\varepsilon_{i j}^{b}$ is background permittivity [64].

Corresponding electric boundary conditions of potential continuity at the mechanically-free particle surface $S$ is $\left.\left(\varphi_{e}-\varphi_{i}\right)\right|_{S}=0$. The boundary condition for the normal components of electric displacements should take into account the surface screening produced by e. g. ambient free charges at the particle surface $S,\left.\left(\left(\mathbf{D}_{e}-\mathbf{D}_{i}\right) \mathbf{n}+\varepsilon_{0} \frac{\varphi_{i}}{\lambda}\right)\right|_{S}=0$, where $\lambda$ is the surface screening length. The potential is constant at the particle-electrode interface, i.e. $\left.\varphi_{i}\right|_{z=0}=0$. Surface screening leads to the decrease of external field inside the particle, as well as to the decrease of bare depolarization field caused by the polarization gradient.

Within a phenomenological approach, linear and biquadratic ME couplings contribution to the system free energy are described by the terms $\mu_{i j} P_{i} M_{j}$ and $\xi_{i j k l} P_{i} P_{j} M_{k} M_{l}$, respectively. $\mathbf{P}$ is polarization and $\mathbf{M}$ is magnetization, and $\mu_{i j}$ and $\xi_{i j k l}$ are corresponding tensors of linear and biquadratic ME effects, respectively [1, 65-68]. The biquadratic ME effect exists in all magnetic materials independently on their magnetic symmetry in contrast to the symmetry-sensitive linear ME effect. The PME coupling contribution is described by the term $\eta_{i j k} P_{i} M_{j} M_{k}$ [13]. As it was shown in Refs. $[13,69]$ the PME coefficient $\eta$ is proportional to the biquadratic ME coefficient $\xi$ that couples the second powers of polarization and magnetic order parameters.

Note that below $T_{N} \approx 650 \mathrm{~K}$ a macroscopic $\mathrm{BiFeO}_{3}$ single-crystal is a G-type canted antiferromagnet with cycloid spin order with a period of about $64 \mathrm{~nm}$ [4, 23]. Canting is caused by Dzialoshinski-Moriya (DM) interaction which becomes to be especially important near the surface of the grains. However the curved surface itself and via the flexomagnetoelectric coupling [70] can affect in unpredictable way the exchange interactions in small spherical $\mathrm{BiFeO}_{3}$ nanoparticles. Moreover the spatial confinement breaks the cycloidal spin order in all directions, where the nanoparticles sizes are less than $100 \mathrm{~nm}$ [37]. The magnetic symmetry changes define the strength 
and symmetry properties of the bilinear magnetoeletric coupling in the $\mathrm{AFM}$ phase of $\mathrm{BiFeO}_{3}$ [71], which in complex becomes an intractable problem. Thus in this work we consider only the paramagnetoelectric (PME) effect that exists even in a paramagnetic phase and, being proportional to the isotropic biquadratic ME coupling coefficient $\xi$, is insensitive to the magnetic symmetry changes in nanoparticles.

Let us use the phenomenological LGD-based model for the PME coefficients calculation [13, 69]. Assuming that the magnetization $\mathrm{M}$ is linearly proportional to the applied magnetic field $\mathrm{H}$, $M \approx \chi_{F M}(T) H$, the PME coefficient $\eta$ has the form [13]:

$$
\eta(T)=-P_{S}(T) \chi_{F E}(T)\left(\chi_{M}(T)\right)^{2} \xi_{M P} .
$$

Here the spontaneous polarization $P_{S}(T)$ is the spontaneous polarization calculated from Eq.(1) at $H=0$ and $E=0$ and averaged over the particle volume, i.e. $P_{S}(T)=\left\langle P_{3}(\mathbf{r})\right\rangle$. Functions $\chi_{M}(T)$ and $\chi_{F E}(T)$ are linear magnetic and dielectric susceptibilities in the ferroelectric phase averaged over the particle volume, respectively. Ferroelectric susceptibility can be calculated from Eq.(1) using the definition

$$
\chi_{F E}(T)=\left.\frac{\partial\left\langle P_{3}\right\rangle}{\partial E_{3}}\right|_{E_{3}=0} .
$$

Approximate expression for magnetic susceptibility is taken the same as in Ref.[13]:

$$
\chi_{M}(T)=\frac{\mu_{0}}{\alpha_{M}^{(T)}(T-\theta)+\xi_{L M} L^{2}+\xi_{M P} P_{S}^{2}(T)} .
$$

Equations (3) and (4) are valid in the FE-AFM phase (with nonzero AFM long-range order parameter $L \neq 0$ ) as well as in the paramagnetic FE phase without any magnetic order at $M=L=0$. Parameters $\xi_{L M}$ and $\xi_{M P}$ are the biquadratic ME coefficients, which couple with the second powers of polarization and magnetic order parameters in the ME energy $G_{M E}=\frac{1}{2}\left(\xi_{M P} M^{2}+\xi_{L P} L^{2}\right) P^{2}$. It should be noted, that only two coefficients in magnetic energy, $G_{M}=\frac{\alpha_{L}(T)}{2} L^{2}+\frac{\beta_{L}}{4} L^{4}+\frac{\alpha_{M}(T)}{2} M^{2}+\frac{\beta_{M}}{4} M^{4}-\mu_{0} M H+\frac{\xi_{L M}}{2} L^{2} M^{2}, \quad$ are $\quad$ assumed $\quad$ to be dependent on temperature, namely $\alpha_{L}(T)=\alpha_{M}^{(T)}\left(T-T_{N}\right)$ and $\alpha_{M}(T)=\alpha_{M}^{(T)}(T-\theta)$ where $\theta$ is the magnetic Curie temperature, and $T_{N}$ is the Neel temperature. 


\subsection{Analytical approximation based on finite element modeling}

Using finite element modeling (FEM) we numerically calculated the spatial distribution [Fig. 2(a)], average electric field properties [Fig. 2(b)-(d)] inside the particle. Parameters of $\mathrm{BiFeO}_{3}$ are listed in Table $\mathbf{1}$.

Table 1. Parameters of $\mathrm{BiFeO}_{3}$ used in our calculations

\begin{tabular}{lcc}
\hline \multicolumn{1}{c}{ Parameter } & SI units & Value for $\mathbf{B i F e O}_{\mathbf{3}}$ \\
\hline Spontaneous polarization $P_{S}$ & $\mathrm{~m} / \mathrm{C}^{2}$ & 1 \\
Electrostriction coefficient $Q_{12}$ & $\mathrm{~m}^{4} / \mathrm{C}^{2}$ & -0.05 \\
Electrostriction coefficient $Q_{11}$ & $\mathrm{~m}^{4} / \mathrm{C}^{2}$ & -0.1 \\
Background permittivity $\varepsilon_{\mathrm{b}}$ & dimensionless & 10 \\
Ambient permittivity $\varepsilon_{\mathrm{e}}$ & dimensionless & 1 \\
Gradient coefficient $\mathrm{g}_{11}$ & $\mathrm{~m}^{3} / \mathrm{F}$ & $10^{-10}$ \\
LGD coefficient $\alpha_{\mathrm{S}}$ & $\mathrm{m}^{2} / \mathrm{F}$ & $10^{-4}$ \\
LGD coefficient $\beta$ & $\mathrm{J} \mathrm{m} / \mathrm{C}^{4}$ & $10^{7}$ \\
LGD coefficient $\alpha$ & $\mathrm{m} / \mathrm{F}$ & $-10^{7}(\mathrm{at} 300 \mathrm{~K})$ \\
Ferroelectric Curie temperature $T_{c}$ & $\mathrm{~K}$ & 1100 \\
Temperature coefficient $\alpha_{T}$ & $\mathrm{~m} /(\mathrm{K} \mathrm{F})$ & $0.9 \times 10^{6}$ \\
Antiferromagnetic Neel temperature & $\mathrm{K}$ & 650 \\
Surface screening length $\lambda$ & $\mathrm{nm}$ & $10^{-3}$ to $10^{2}$ \\
Universal dielectric constant $\varepsilon_{0}$ & $\mathrm{~F} / \mathrm{m}$ & $8.85 \times 10^{-12}$ \\
\hline
\end{tabular}

Numerical results were approximated analytically. Obtained electric field dependence on the surface screening length $\lambda$ has the following form:

$$
E_{d X} \approx-\frac{P_{X}}{\varepsilon_{0}} \frac{\lambda n_{\infty}(a, b, c)}{\lambda+R n_{\infty}(a, b, c)}
$$

Here $n_{\infty}$ is the "bare" depolarization factor of the system without screening charges (the case of the limit $\lambda \rightarrow \infty)$. $R$ is the characteristic length-scale, proportional to the size $a$ of island along the polar axis. Using Eq.(6) we can introduce the effective depolarization factor $n_{d}(a, b, c)=-\varepsilon_{0} \frac{E_{d X}}{P_{X}}$ that is dependent on the semi-ellipsoid geometry as

$$
n_{d}(a, b, c)=\frac{\lambda n_{\infty}(a, b, c)}{\lambda+R n_{\infty}(a, b, c)}
$$

High accuracy of approximation (7) becomes evident from Fig. 2(b).

The fitting with Eq.(7) allows us to obtain the parameters $n_{\infty}$ and $R$ for a set of island sizes $a, b$ and $c$, which are the lengths of ellipsoid semi-axis. These sets were fitted with the Padeapproximations of the following form: 


$$
\begin{gathered}
n_{\infty}(a, b, c) \approx \frac{b}{\varepsilon_{b} b+\varepsilon_{e} a}\left(\frac{c^{2}}{c^{2}+0.7 a c+a^{2} \frac{b}{b+0.075 a}}\right), \\
R(a, b, c) \approx a\left(0.62+0.19 \frac{a}{b}+0.25 \frac{a}{c}\right) .
\end{gathered}
$$

Note that the pre-factor $\frac{b}{\varepsilon_{b} b+\varepsilon_{e} a}$ in Eq.(8) is the exact expression for depolarization factor of elliptical cylinder with semi-axes $a$ and $b$. High accuracy of approximations (8)-(9) becomes evident from Figs. 2(b)-(d).

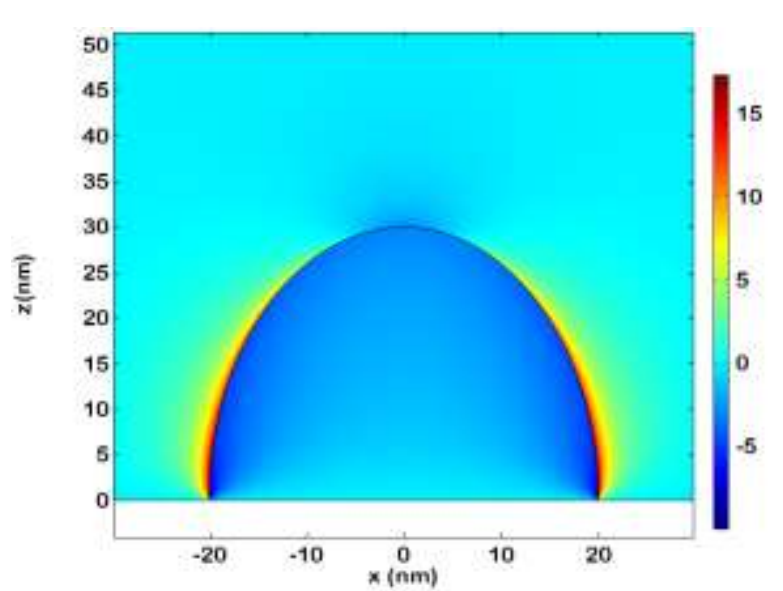

(a)
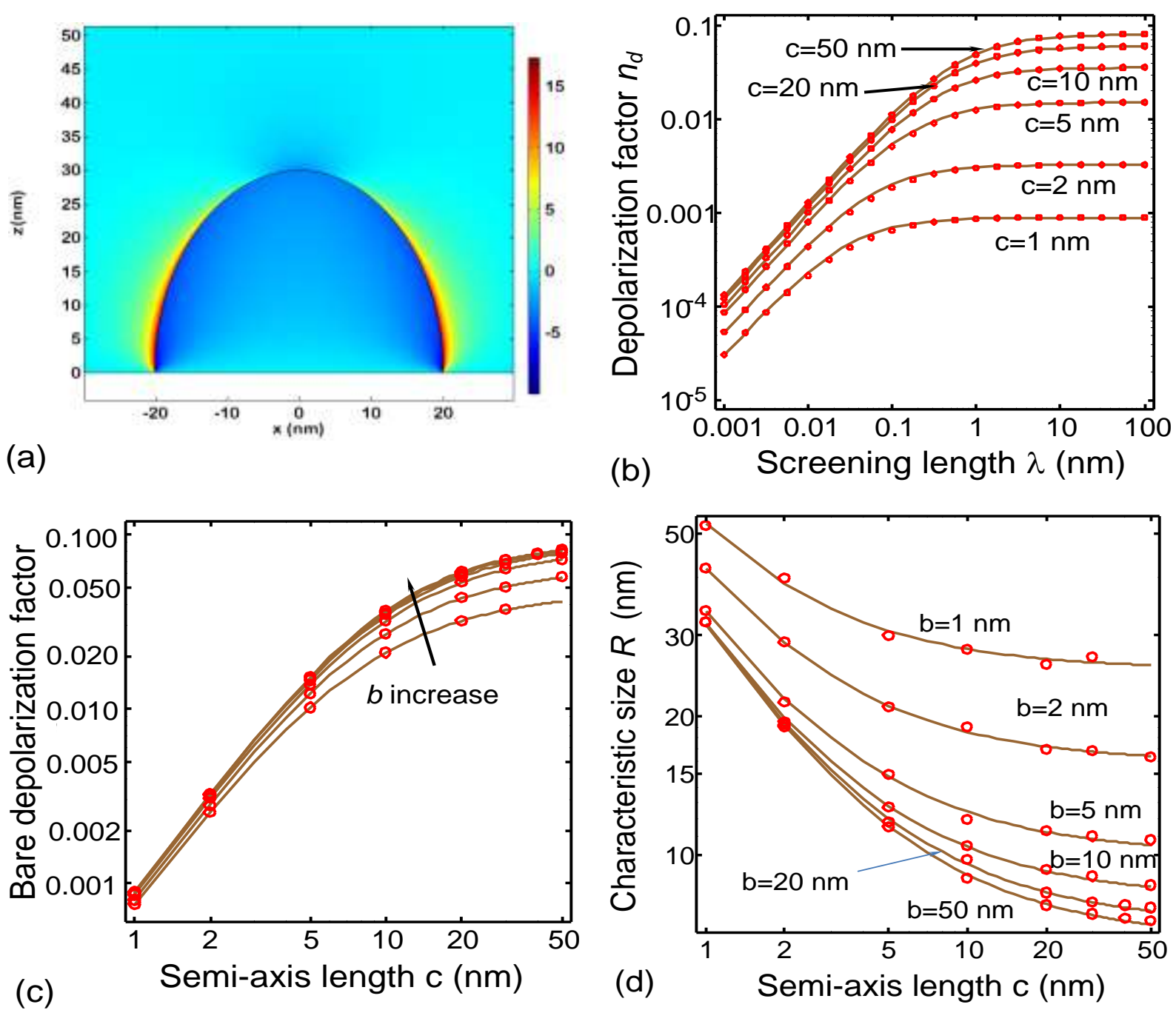

Fig. 2. (a) Distribution of the electric field component $E_{x}$ in the system calculated numerically. (b) Dependence of depolarization factor $n_{X}$ on the screening length for different values of semi-axis length $c$ (the numbers are specified near the curves) and fixed value of $a=10 \mathrm{~nm}$ and $b=20 \mathrm{~nm}$. Dependences of parameters $n_{\infty}$ (c) and $R$ (d) on the semi-axis length $c$ for fixed values of $a=10 \mathrm{~nm}$ and $b=1,2,5,10,20,50$ 
$\mathrm{nm}$ (the numbers are specified near the curves). Relative dielectric permittivities $\varepsilon_{b}=10$ and $\varepsilon_{e}=1$. Symbols are results of FEM calculations, solid curves represent fitting with Eq.(6) - (9), respectively.

Allowing for expressions (6)-(9), the transition temperature to paraelectric phase $T_{c r}(a, b, c)$ can be defined from the condition $\alpha+\frac{n_{d}}{\varepsilon_{0}}=0$ and is given by analytical expression

$$
T_{c r}(a, b, c)=T_{C}-\frac{n_{d}(a, b, c)}{\alpha_{T} \varepsilon_{0}} .
$$

Equation (10) allows writing analytical expressions for the average spontaneous polarization and linear dielectric susceptibility by conventional form

$$
\begin{gathered}
P_{S}= \begin{cases}\sqrt{\frac{\alpha_{T}}{\beta}\left(T_{c r}(a, b, c)-T\right)}, & T<T_{c r}, \\
0, \quad T>T_{c r} .\end{cases} \\
\chi_{F E}(T)= \begin{cases}\frac{1}{2 \alpha_{T}\left(T_{c r}(a, b, c)-T\right)}, & T<T_{c r}, \\
\frac{1}{\alpha_{T}\left(T-T_{c r}(a, b, c)\right)}, & T>T_{c r} .\end{cases}
\end{gathered}
$$

Allowing for Eqs.(3), (5), (11) and (12) the analytical expression for PME coefficient acquires the form:

$$
\eta(T)=\left\{\begin{array}{l}
\frac{-\xi_{M P}\left(\chi_{M}(T)\right)^{2}}{2 \sqrt{\alpha_{T} \beta\left(T_{c r}(a, b, c)-T\right)}}, \quad T<T_{c r}, \\
0, \quad T>T_{c r} .
\end{array}\right.
$$

It follows from the obtained formula (10) - (13), that the main peculiarities of the semi-ellipsoidal nanoparticles originate from the depolarization field contribution.

\section{Results and discussion}

\subsection{Size effects of phase diagrams and average polarization}

Phase diagrams of semi-ellipsoidal $\mathrm{BiFeO}_{3}$ nanoparticles in coordinates "relative temperature $T / T_{C}$ - length of the particle longer semi-axis $a^{\prime \prime}$ are shown in Figs.3(a) and 3(b). The boundary between paraelectric (PE) and ferroelectric (FE) phases (that is in fact the critical temperature of the size-induced phase transition $\left.T_{c r}(a, b, c)\right)$ depends on the semi-ellipsoid sizes $\mathrm{a}, \mathrm{b}$ and c (multiple size effect). The size effect manifesting itself in the ferroelectricity disappearance at the critical size $a_{c r}(b, c)$ for which $T_{c r}=0$, is followed by the monotonic increase of the transition temperature with the $a$-size increase and its further saturation to $T_{C}$ for the sizes $a>100 \mathrm{~nm}$. 
Different curves are calculated for several values of semi-axis $b=3,10,30$ and $100 \mathrm{~nm}$ and fixed particle height $c$. Figure 3(a) corresponds to the particles of small height $c=10 \mathrm{~nm}$ and Fig.3(b) corresponds to $c=100 \mathrm{~nm}$. The critical size $a_{c r}(b, c)$ monotonically decreases and the phase boundary between PE and FE phases shifts to the right with $b$ increase at the same $c$ values [compare different curves in Figs. 3(a) and 3(b)]. Moreover, the critical sizes $a_{c r}(b, c)$ calculated at $c=10 \mathrm{~nm}$ are essentially smaller than the sizes calculated at $c=100 \mathrm{~nm}$ at the same $b$-values [compare the curves in Figs. 3(a) and 3(b)]. In numbers, the PE-FE transition exists for all values of chosen sizes. At $c=10 \mathrm{~nm}$, the critical size $a_{c r}(b, c)$ varies in the narrow range $(10-12) \mathrm{nm}$, and the curves calculated for different $b$ values are very close to one another. At $c=100 \mathrm{~nm}$, the critical size $a_{c r}(b, c)$ varies in the wider range $(15-45) \mathrm{nm}$, and the curves calculated for different $b$ values are well separated from each other.

Hence the analysis of Figs. 3(a) and 3(b) allows us to conclude that the size effect of the phase diagrams is sensitive to the aspect ratio of the particle sizes in the polarization direction, $b c / a^{2}$, and less sensitive to the absolute values of the sizes. The smaller the ratio, the smaller the depolarization field and hence the higher the transition temperature and the smaller the critical size. The result seems to be nontrivial a priori.

The spontaneous polarization dependence on the length of ellipsoid semi-axis $a$ calculated for different values of semi-axis $c=10 \mathrm{~nm}$ and $100 \mathrm{~nm}$ at room temperature are shown in Figs. 3(c) and 3(d), respectively. The values of another semi-axis $b$ are chosen the same as in Figs. 3(a) and 3(b) [see different curves calculated for $b=3,10,30$ and $100 \mathrm{~nm}$ ]. The polarization curves calculated for different $b$ values are very close to one another at $c=10 \mathrm{~nm}$, and are well-separated from one another at $c=100 \mathrm{~nm}$. The spontaneous polarization appears at the critical size $a_{c r}(b, c)$ and increases with the size $a$ increase. The polarization saturates to the bulk value $\sim 1 \mathrm{C} / \mathrm{m}^{2}$ at sizes $a>100 \mathrm{~nm}$. Note that the polarization of the particles with height $c=10 \mathrm{~nm}$ saturates essentially faster than the one for the particles with $c=100 \mathrm{~nm}$ [compare curves saturation in Figs. 3(c) and 3(d)]. 


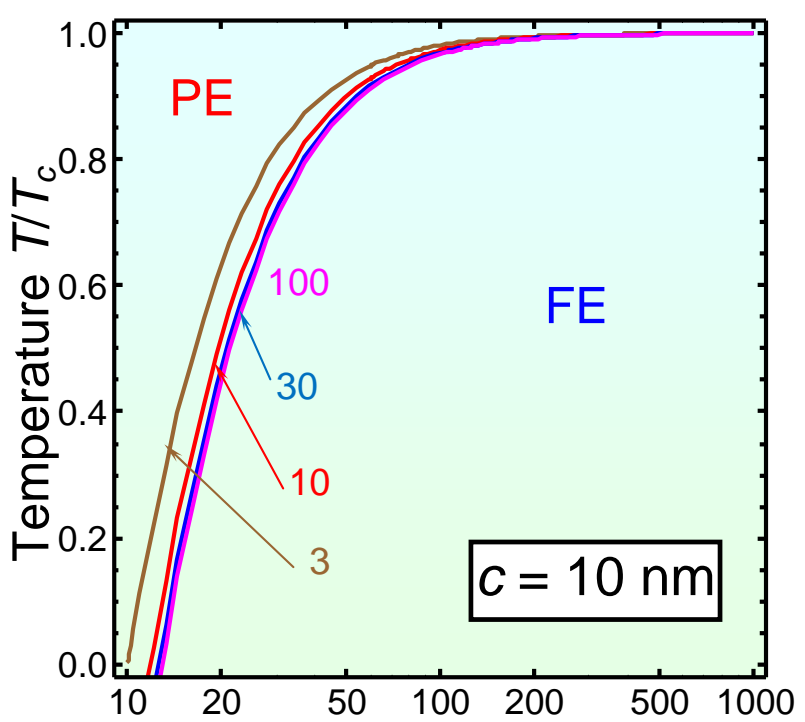

(a)

Semi-axis a $(\mathrm{nm})$

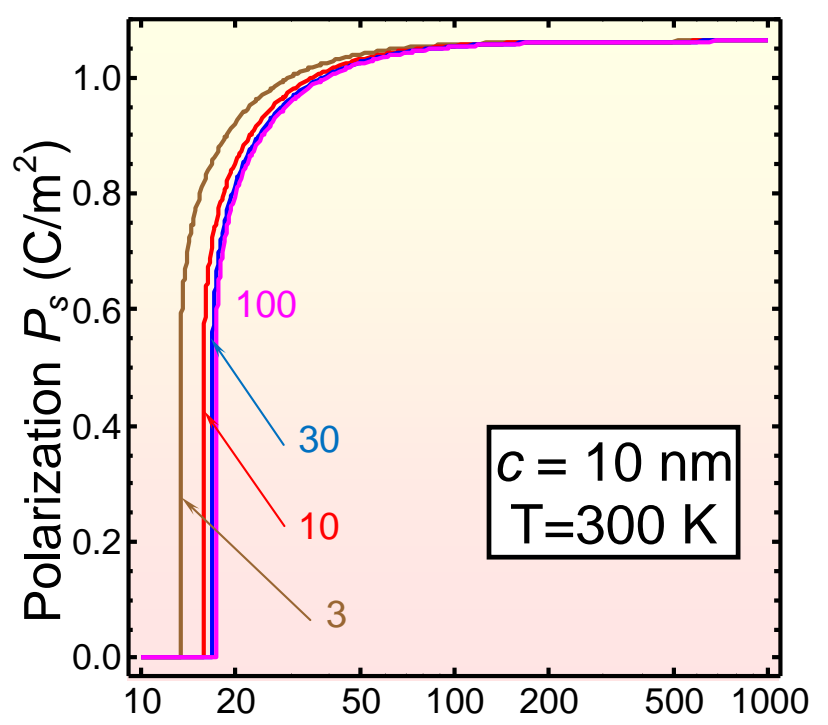

(c)

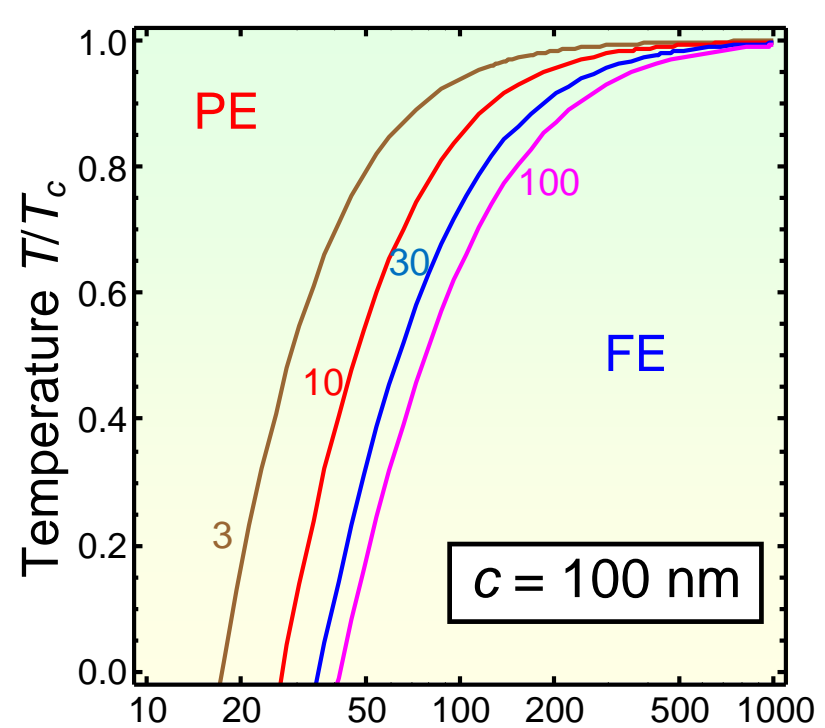

(b)

Semi-axis a $(\mathrm{nm})$

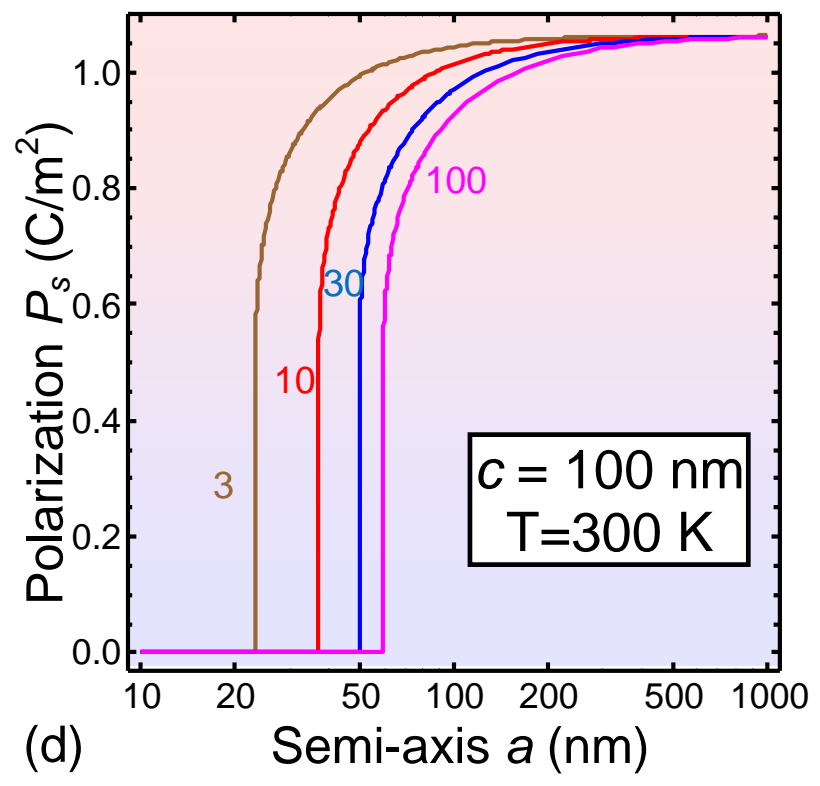

Fig. 3. Phase diagrams in coordinates "temperature - length of ellipsoid semi-axis $a$ " calculated for different values of semi-axis $c=10 \mathrm{~nm}$ (a) and $100 \mathrm{~nm}$ (b) and axis b=3, 10, 30 and $100 \mathrm{~nm}$ (specified by numbers near the curves). The dependence of spontaneous polarization on the length of ellipsoid semi-axis $a$ calculated at room temperature for different values of semi-axis $c=10 \mathrm{~nm}$ (c) and $100 \mathrm{~nm}$ (d) and axis $b=3$, 10, 30 and $100 \mathrm{~nm}$ (specified by numbers near the curves). Screening length $\lambda=1 \mathrm{~nm}$, other parameters corresponding to $\mathrm{BiFeO}_{3}$ compound are listed in Table 1 .

\subsection{Size effect of paramagnetoelectric coefficient}

The dependences of PME effect coefficient on the length of ellipsoid semi-axis $a$ calculated at room temperature for different values of the semi-axis $c=10 \mathrm{~nm}$ and $100 \mathrm{~nm}$ are shown in Figs. 4(a) and 4(b), respectively. The values of another semi-axis $b$ are chosen the same as in the previous figures [see different curves calculated for $b=3,10,30$ and $100 \mathrm{~nm}$ ]. PME coefficient is 
normalized on its bulk value. The PME coefficient is zero at sizes $a<a_{c r}(b, c)$ because of spontaneous polarization disappearance, it appears at $a<a_{c r}$ and diverges at the critical size $a=a_{c r}(b, c)$, and then it decreases with the size $a$ increase. The PME coefficient saturates to the bulk value at sizes $a>100 \mathrm{~nm}$. The divergences at $a=a_{c r}(b, c)$ demonstrate the possibility to obtain giant $\mathrm{PME}$ effect in $\mathrm{BiFeO}_{3}$ nanoparticles in the vicinity of size-induced transition from the FE phase to a PE phase. In particular the normalized PME coefficient is essentially higher than unity for sizes $a_{c r}(b, c) \leq a<2 a_{c r}(b, c)$. The behavior of PME coefficient reproduces the behavior of the dielectric susceptibility given by Eq.(12) in the framework of our model. Note that the PME coefficient of the particles with height $c=10 \mathrm{~nm}$ saturates essentially faster than the one for the particles with $c=100 \mathrm{~nm}$ [compare curves saturation in Figs. 4(a) and 4(b)]. The PME coefficient curves calculated for different $b$ values are very close to one another at $c=10 \mathrm{~nm}$, and are wellseparated from one another at $c=100 \mathrm{~nm}$.

The comparative analyses of the Figs.3(c)-(d) and Figs.4(a)-(b) approves our conclusion that the size effect of the spontaneous polarization and PME coefficient is sensitive to the aspect ratio of particle sizes in the polarization direction, $b c / a^{2}$, and less sensitive to the absolute values of the sizes.
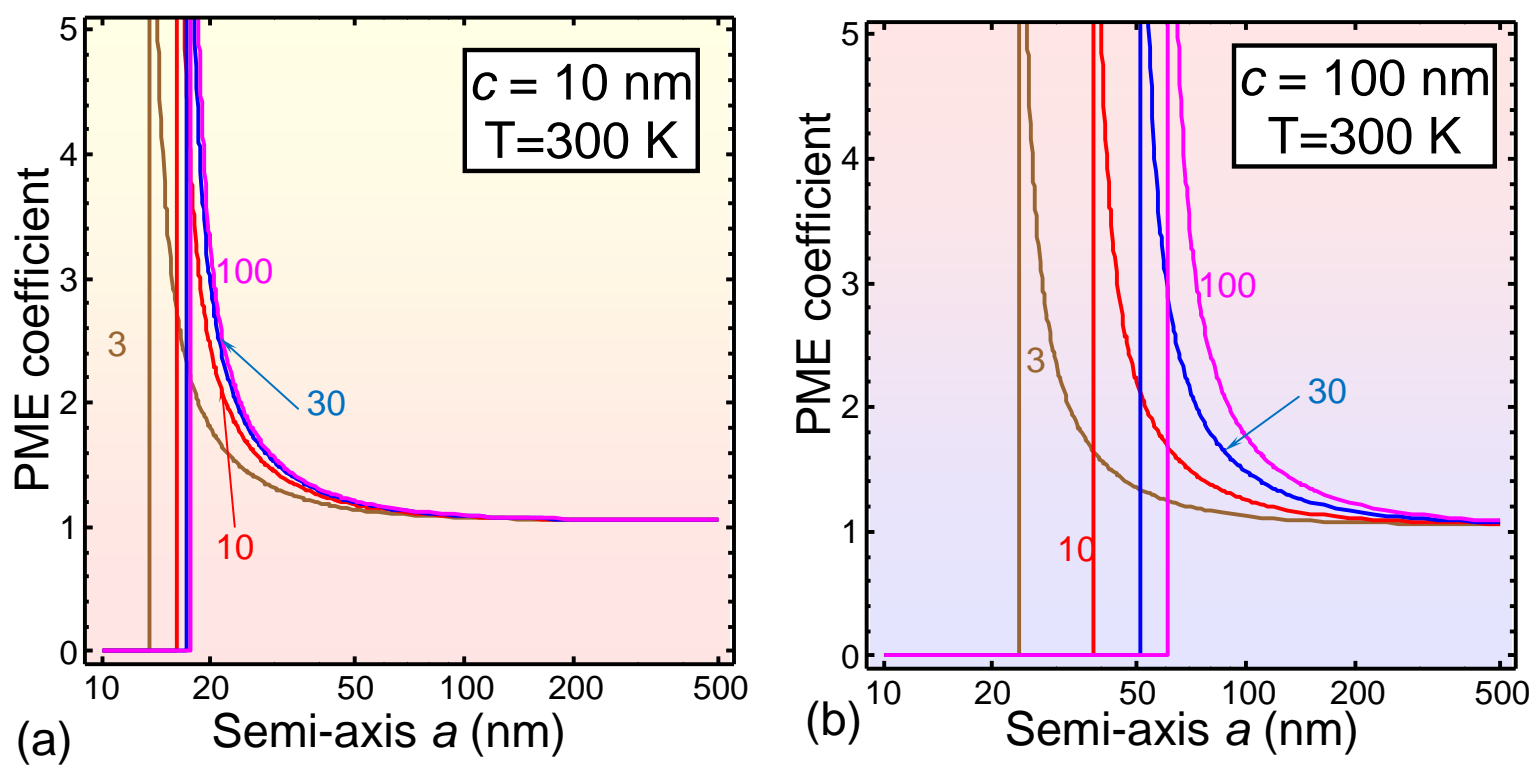

Fig. 4. Dependence of the normalized PME effect coefficient on the length of semi-ellipsoid semi-axis $a$ calculated for different values of semi-axis c=10 $\mathrm{nm}$ and $100 \mathrm{~nm}$ (panels (a) and (b), respectively). Different curves correspond to the different values of the axis $b=3,10,30$ and $100 \mathrm{~nm}$ (specified by numbers near the curves). Screening length $\lambda=1 \mathrm{~nm}$, room temperature, other parameters corresponding to $\mathrm{BiFeO}_{3}$ compound are listed in Table 1. 


\section{Conclusions}

We have studied the size effects on the phase diagrams, ferroelectric and magnetoelectric properties of semi-ellipsoidal $\mathrm{BiFeO}_{3}$ nanoparticles clamped to a rigid conductive substrate. The spatial distribution of the spontaneous polarization vector inside the ferroelectric nanoparticles, phase diagrams and paramagnetoelectric coefficient were calculated in the framework of the Landau-Ginzburg-Devonshire approach, classical electrostatics and elasticity theory. Rather rigorous analytical expressions were derived for the dependences of the ferroelectric transition temperature, average polarization, linear dielectric susceptibility and paramagnetoelectric coefficient on the particle sizes for a general case of a semi-ellipsoidal nanoparticles with different semi-axes $a, b$ and height $c$. The in-plane orientation of the spontaneous polarization along the longer semi-ellipsoidal axis $a$ is energetically more preferable for the nanoparticles of small height $c<a$ due to the essential decrease of depolarization field. The analyses of the obtained results leads to the conclusion that the size effect on the phase diagrams, spontaneous polarization and paramagnetoelectric coefficient is rather sensitive to the aspect ratio of particle sizes in the polarization direction, $b c / a^{2}$, and less sensitive to the absolute values of the sizes. This opens the way to govern the properties by the choice of this ratio value.

Acknowledgments. M.V.S. and D.V.K acknowledge MK-1720.2017.8, BRFFI (\# F16R-066) RFFI (\# 1658-00082). E.A.E. and A.N.M. acknowledge the Center for Nanophase Materials Sciences, which is a DOE Office of Science User Facility, CNMS2016-061.

Authors' contribution. A.N.M. generated the research idea, states the mathematical problem and wrote the manuscript draft. V.V.K. and E.A.E. performed numerical simulations. E.A.E. and A.N.M. derived analytical expressions. M.D.G. worked on the results interpretation and manuscript improvement. M.V.S. and D.V.K. valuably contributed to the research idea and manuscript improvement.

\section{References}

[1] M. Fiebig, Revival of the magnetoelectric effect, J. Phys. D: Appl. Phys. 38 (2005) 123-152.

[2] N. A. Spaldin and M. Fiebig, Materials science. The renaissance of magnetoelectric multiferroics, Science 309 (2005) 391-392.

[3] J.M. Rondinelli, N.A. Spaldin, Structure and properties of functional oxide thin films: Insights from electronicstructure calculations, Adv. Mater. 23 (2011) 3363-3381.

[4] A. P. Pyatakov, A. K.Zvezdin, Magnetoelectric and multiferroic media, Physics-Uspekhi 55:6 (2012) $557-581$.

[5] J. F. Scott, Data storage: Multiferroic memories, Nat. Mater. 6 (2007) 256-257.

[6] R. Ramesh \& A. Nicola Spaldin, Multiferroics: progress and prospects in thin films, Nat. Mater. 6 (2007) 21-29. 
[7] P.J. Ryan, J-W. Kim, T. Birol, P. Thompson, J-H. Lee, X. Ke, P.S. Normile, E. Karapetrova, P. Schiffer, S.D. Brown, C.J. Fennie, D.G. Schlom, Reversible control of magnetic interactions by electric field in a single-phase material, Nat. Commun. 4 (2013) 1334.

[8] M.J. Haun, E. Furman, T.R. Halemane, L.E. Cross, Thermodynamic theory of the lead zirconate-titanate solid solution system, part IV: tilting of the oxygen octahedral, Ferroelectrics 99 (1989) 55-62.

[9] A.K. Tagantsev, E. Courtens, L. Arzel, Prediction of a low-temperature ferroelectric instability in antiphase domain boundaries of strontium titanate, Phys. Rev. B 64 (2001) 224107.

[10] S.L. Hou, N. Bloembergen, Paramagnetoelectric effects in $\mathrm{NiSO}_{4} \cdot 6 \mathrm{H}_{2} \mathrm{O}$, Phys. Rev. 138 (1965) 1218-1226.

[11] V.V. Shvartsman, S. Bedanta, P. Borisov, W. Kleemann, (Sr,Mn)TiO ${ }_{3}$ : A magnetoelectric multiglass, Phys. Rev. Lett. 101 (2008) 165704.

[12] W. Kleemann, V.V. Shvartsman, P. Borisov, A. Kania, Coexistence of antiferromagnetic and spin cluster glass order in the magnetoelectric relaxor multiferroic $\mathrm{PbFe}_{0.5} \mathrm{Nb}_{0.5} \mathrm{O}_{3}$, Phys. Rev. Lett. 105 (2010) 257202.

[13] V. V. Laguta, A. N. Morozovska, E. A. Eliseev, I. P. Raevski, S. I. Raevskaya, E. I. Sitalo, S. A. Prosandeev, and L. Bellaiche,. Room-temperature paramagnetoelectric effect in magnetoelectric multiferroics $\mathrm{Pb}\left(\mathrm{Fe}_{1 / 2} \mathrm{Nb}_{1 / 2}\right) \mathrm{O}_{3}$ and its solid solution with $\mathrm{PbTiO}_{3}$, J. Mater. Sci. 51 (2016) 5330-5342.

[14] Y.-H. Chu, L.W. Martin, M.B. Holcomb, M. Gajek, S.-J. Han, Q.He, N.Balke, C.-H. Yang, D. Lee, W. Hu, Q. Zhan, P.-L. Yang, A. Fraile-Rodríguez, A. Scholl, S.X. Wang and R. Ramesh, Electric-field control of local ferromagnetism using a magnetoelectric multiferroic, Nat. Mater. 7 (2008) 478 - 482.

[15] C.-S. Chen, C.-S. Tu, P.-Y. Chen, V. H. Schmidt, Z.-R. Xu, and Y. Ting, Spin-lattice coupling phase transition and phonon anomalies in bismuth ferrite $\mathrm{BiFeO}_{3}$, J. Alloys Compd. 687 (2016) 442-450.

[16] J. Seidel, L.W. Martin, Q. He, Q. Zhan, Y.-H. Chu, A. Rother, M. E. Hawkridge, P. Maksymovych, P. Yu, M. Gajek, N. Balke, S. V. Kalinin, S. Gemming, F. Wang, G. Catalan, J. F. Scott, N. A. Spaldin, J. Orenstein and R. Ramesh, Conduction at domain walls in oxide multiferroics, Nat. Mater. 8 (2009) 229 - 234.

[17] J. Seidel, P. Maksymovych, Y. Batra, A. Katan, S.-Y. Yang, Q. He, A. P. Baddorf, S. V. Kalinin, C.-H.Yang, J.-C. Yang, Y.-H. Chu, E. K. H. Salje, H.Wormeester, M. Salmeron and R. Ramesh, Domain wall conductivity in La-doped $\mathrm{BiFeO}_{3}$, Phys. Rev. Lett. 105 (2010) 197603.

[18] Q. He, C.-H. Yeh, J.-C. Yang, G. Singh-Bhalla, C.-W. Liang, P.-W. Chiu, G. Catalan, L.W. Martin, Y.-H. Chu, J. F. Scott, and R. Ramesh, Magnetotransport at domain walls in $\mathrm{BiFeO}_{3}$, Phys. Rev. Lett. 108 (2012) 067203.

[19] G. Catalan, J. Seidel, R. Ramesh, and J. F. Scott, Domain wall nanoelectronics, Rev. Mod. Phys. 84 (2012) 119. [20] R. K. Vasudevan, A. N. Morozovska, E. A. Eliseev, J. Britson, J.-C. Yang, Y.-H. Chu, P. Maksymovych, L. Q. Chen, V. Nagarajan, S. V. Kalinin. Domain wall geometry controls conduction in ferroelectrics, Nano Lett. 12 (11) (2012) 5524-5531.

[21] A. N. Morozovska, Rama K. Vasudevan, P. Maksymovych, S. V. Kalinin, and E. A. Eliseev, Anisotropic conductivity of uncharged domain walls in $\mathrm{BiFeO}_{3}$, Phys. Rev. B. 86 (2012) 085315.

[22] P. Fischer, M. Polomska, I. Sosnowska, M. Szymanski, Temperature dependence of the crystal and magnetic structures of $\mathrm{BiFeO}_{3}$, J. Phys. C: Solid St. Phys. 13 (1980) 1931-1940.

[23] G. Catalan, J.F. Scott, Physics and applications of bismuth ferrite, Adv. Mater. 21 (2009) 1-23.

[24] Y.-H. Chu, Qian Zhan, Lane W. Martin, Maria P. Cruz, Pei-Ling Yang, Gary W. Pabst, Florin Zavaliche, SeungYeul Yang, Jing-Xian Zhang, Long-Qing Chen, Darrell G. Schlom, I.-Nan Lin, Tai-Bor Wu, and Ramamoorthy Ramesh, Nanoscale domain control in multiferroic $\mathrm{BiFeO}_{3}$ thin films, Adv. Mater. 18 (2006) 2307. 
[25] P. Maksymovych, M. Huijben, Minghu Pan, S. Jesse, N.a Balke, Ying-Hao Chu, Hye Jung Chang, A. Y. Borisevich, A. P. Baddorf, G. Rijnders, D.E.H.A. Blank, R. Ramesh, and S. V. Kalinin, Ultrathin limit and dead-layer effects in local polarization switching of $\mathrm{BiFeO}_{3}$, Phys. Rev. B 85 (2012) 014119.

[26] C. Beekman, W. Siemons, M. Chi, N. Balke, J. Y. Howe, T. Z. Ward, P. Maksymovych, J. D. Budai, J. Z. Tischler, R. Xu, W. Liu, and H. M. Christen., Ferroelectric self-poling, switching, and monoclinic domain configuration in $\mathrm{BiFeO}_{3}$,Thin Films, Adv. Funct. Mater. 26 (2016) 5166-5173.

[27] N. Price, Waterfield, R. D. Johnson, W. Saenrang, F. Maccherozzi, S. S. Dhesi, A. Bombardi, F. P. Chmiel, C-B. Eom, and P. G. Radaelli, Coherent magnetoelastic domains in multiferroic $\mathrm{BiFeO}_{3}$ films, Phys. Rev. Lett. 117 (2016) 177601 .

[28] J. Wang, J. B. Neaton, H. Zheng, V. Nagarajan, S. B. Ogale, B. Liu, D. Viehland, V. Vaithyanathan, D. G. Schlom, U. V. Waghmare, N. A. Spaldin, K. M. Rabe, M. Wuttig, R. Ramesh, Epitaxial $\mathrm{BiFeO}_{3}$ multiferroic thin film heterostructures, Science 299 (2003) 1719.

[29] A.Y. Borisevich, O.S. Ovchinnikov, Hye Jung Chang, M.P. Oxley, Pu Yu, Jan Seidel, E.A. Eliseev, A.N. Morozovska, Ramamoorthy Ramesh, S.J. Pennycook, S.V. Kalinin., Beyond condensed matter physics on the nanoscale: the role of ionic and electrochemical phenomena in the physical functionalities of oxide materials, ACS Nano 4 (2010) 6071-6079.

[30] N. Balke, B. Winchester, Wei Ren, Ying Hao Chu, A. N. Morozovska, E. A. Eliseev, M. Huijben, Rama K. Vasudevan, P. Maksymovych, J. Britson, S. Jesse, I. Kornev, Ramamoorthy Ramesh, L. Bellaiche, Long Qing Chen, and S. V. Kalinin, Enhanced electric conductivity at ferroelectric vortex cores in $\mathrm{BiFeO}_{3}$, Nature Physics 8 (2012) 8188.

[31] Y.-M. Kim, A. Kumar, A. Hatt, A. N. Morozovska, A. Tselev, M. D. Biegalski, I. Ivanov, E. A. Eliseev, S. J. Pennycook, J. M. Rondinelli, S. V. Kalinin, A. Y. Borisevich, Interplay of octahedral tilts and polar order in $\mathrm{BiFeO}_{3}$ films, Adv. Mater. 25 (2013) 2497-2504.

[32] R. K. Vasudevan, W. Wu, J. R. Guest, A. P. Baddorf, A. N. Morozovska, E. A. Eliseev, N. Balke, V. Nagarajan, P. Maksymovych, Domain wall conduction and polarization-mediated transport in ferroelectrics, Adv. Funct. Mater. 23 (2013) 2592-2616.

[33] Y.-M. Kim, A. Morozovska, E. Eliseev, M. Oxley, R. Mishra, T. Grande, S. Selbach, S. Pantelides, S. Kalinin, and A. Borisevich, Direct observation of ferroelectric field effect and vacancy-controlled screening at the $\mathrm{BiFeO}_{3}-\mathrm{La}_{\mathrm{x}} \mathrm{Sr}_{1-}$ ${ }_{\mathrm{x}} \mathrm{MnO}_{3}$ interface, Nat. Mater. 13 (2014) 1019-1025.

[34] B. Winchester, N. Balke, X. X. Cheng, A. N. Morozovska, S. Kalinin, and L. Q. Chen, Electroelastic fields in artificially created vortex cores in epitaxial $\mathrm{BiFeO}_{3}$ thin films, Appl. Phys. Lett. 107 (2015) 052903.

[35] J. F. Scott, Advanced Materials, Iso-structural phase transitions in $\mathrm{BiFeO}_{3}$ Adv. Mater. 22 (2010) 2106-2107.

[36] S. Layek and H. C. Verma, Magnetic and dielectric properties of multiferroic $\mathrm{BiFeO}_{3}$ nanoparticles synthesized by a novel citrate combustion method, Adv. Mat. Lett. 3 (2012) 533-538.

[37] F. Huang, Z. Wang, X. Lu, J. Zhang, K. Min, W. Lin, R. Ti, Ting-Ting Xu, Ju He, Chen Yue \& J. Zhu, Magnetism of $\mathrm{BiFeO}_{3}$ nanoparticles peculiar with size approaching the period of the spiral spin structure, Sci. Rep. 3 (2013) 2907. [38] D. Yadlovker, S. Berger, Uniform orientation and size of ferroelectric domains, Phys. Rev. B. 71 (2005) 184112. [39] Z. Zhao, V. Buscaglia, M. Viviani, M.T. Buscaglia, L. Mitoseriu, A. Testino, M. Nygren, M. Johnsson, P. Nanni, Grain-size effects on the ferroelectric behavior of dense nanocrystalline $\mathrm{BaTiO}_{3}$ ceramics, Phys. Rev. B 70 (2004) 024107. 
[40] E. Erdem, H.-Ch. Semmelhack, R. Bottcher, H. Rumpf, J. Banys, A.Matthes, H.-J. Glasel, D. Hirsch, E. Hartmann, Study of the tetragonal-to-cubic phase transition in $\mathrm{PbTiO}_{3}$ nanopowders, J. Phys.: Condens. Matter 18 (2006) 38613874.

[41] I.S. Golovina, S.P. Kolesnik, V. Bryksa, V.V. Strelchuk, I.B. Yanchuk, I.N. Geifman, S.A. Khainakov, S.V.

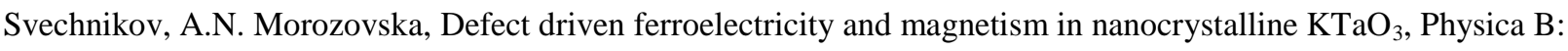
Cond. Matter. 407 (2012) 614-623.

[42] T. Yu, Z. X. Shen, W. S. Toh, J. M. Xue, J. Wang, Size effect on the ferroelectric phase transition in $\mathrm{SrBi}_{2} \mathrm{Ta}_{2} \mathrm{O}_{9}$ nanoparticles, J. Appl. Phys. 94 (2003) 618.

[43] H. Ke, D. C. Jia, W. Wang, Y. Zhou, Ferroelectric phase transition investigated by thermal analysis and Raman scattering in $\mathrm{SrBi}_{2} \mathrm{Ta}_{2} \mathrm{O}_{9}$ nanoparticles, Solid State Phenomena 121-123 (2007) 843-846.

[44] V. Annapu Reddy, N. P. Pathak, and R. Nath, Particle size dependent magnetic properties and phase transitions in multiferroic $\mathrm{BiFeO}_{3}$ nano-particles, J. Alloys Compd. 543, (2012) 206

[45] C. Peng, X. Xu, C. Koenigsmann, A.C. Santulli, S.S. Wong, and J.L. Musfeldt, Size-dependent infrared phonon modes and ferroelectric phase transition in $\mathrm{BiFeO}_{3}$ nanoparticles, Nano Lett. 10, (2010) 4526.

[46] X. Bai, J. Wei, B. Tian, Y. Liu, T. Reiss, N. Guiblin, P. Gemeiner, B. Dkhil, and I.C. Infante, Size effect on optical and photocatalytic properties in $\mathrm{BiFeO}_{3}$ nanoparticles, J. Phys. Chem. C 120 (2016) 3595-3601.

[47] J. Hoon Jeon, H.-Y. Joo, Y.-M. Kim, D. Hyun Lee, J.-S.Kim, Y.S. Kim, T.Choi, and B.H. Park, Selector-free resistive switching memory cell based on $\mathrm{BiFeO}_{3}$ nano-island showing high resistance ratio and nonlinearity factor, Sci. Rep. 6 (2016) 23299.

[48] M. Hasan, M. A. Basith, M. A. Zubair, M. S.Hossain, R. Mahbub, M. A. Hakim, and M. F. Islam, Saturation magnetization and band gap tuning in $\mathrm{BiFeO}_{3}$ nanoparticles via co-substitution of Gd and Mn, J. Alloys Compd. 687 (2016) 701-706.

[49] T.Wang, T.Xu, S.Gao, and S-H. Song, Effect of Nd and Nb co-doping on the structural, magnetic and optical properties of multiferroic $\mathrm{BiFeO}_{3}$ nanoparticles prepared by sol-gel method. Cer. Intern. 43 (2017) 4489-4495. [50] P.P. Khirade, S.D. Birajdar, A. B. Shinde, and K. M. Jadhav. Room temperature ferromagnetism and photoluminescence of multifunctional Fe doped $\mathrm{BaZrO}_{3}$ nanoceramics, J. Alloys Compd. 691 (2017) 287-298. [51] P. Perriat, J. C. Niepce, G. Caboche, Thermodynamic considerations of the grain size dependency of material properties: a new approach to explain the variation of the dielectric permittivity of $\mathrm{BaTiO}_{3}$ with grain size, J. Therm. Anal. Calorim. 41 (1994) 635-649.

[52] H. Huang, C. Q. Sun, P. Hing, Surface bond contraction and its effect on the nanometric sized lead zirconate titanate, J. Phys.: Condens. Matter 12 (2000) 127-132.

[53] H. Huang, C. Q. Sun, Z. Tianshu, P. Hing, Grain-size effect on ferroelectric $\mathrm{Pb}\left(\mathrm{Zr}_{1-\mathrm{x}} \mathrm{Ti}_{\mathrm{x}}\right) \mathrm{O}_{3}$ solid solutions induced by surface bond contraction, Phys. Rev. B 63 (2001) 184112.

[54] M. Wenhui, Surface tension and Curie temperature in ferroelectric nanowires and nanodots, Appl. Phys. A 96 (2009) 915-920.

[55] E.A. Eliseev, A.N. Morozovska, M.D. Glinchuk, R. Blinc, Spontaneous flexoelectric/flexomagnetic effect in nanoferroics, Phys. Rev. B. 79 (2009) 165433.

[56] A. N. Morozovska, E. A. Eliseev, M.D. Glinchuk, Ferroelectricity enhancement in confined nanorods: Direct variational method, Phys. Rev. B 73 (2006) 214106.

[57] A. N. Morozovska, M. D. Glinchuk, E.A. Eliseev, Phase transitions induced by confinement of ferroic nanoparticles, Phys. Rev. B 76 (2007) 014102. 
[58] A.N. Morozovska, I.S. Golovina, S.V. Lemishko, A.A. Andriiko, S.A. Khainakov, E.A. Eliseev, Effect of Vegard strains on the extrinsic size effects in ferroelectric nanoparticles, Phys. Rev. B 90 (2014) 214103.

[59] A. N. Morozovska, and M. D. Glinchuk, Reentrant phase in nanoferroics induced by the flexoelectric and Vegard effects, J. Appl. Phys. 119 (2016) 094109.

[60] E.A. Eliseev, A.V. Semchenko, Y.M. Fomichov, M. D. Glinchuk, V.V. Sidsky, V.V. Kolos, Yu.M. Pleskachevsky, M.V. Silibin, N.V. Morozovsky, A.N. Morozovska, Surface and finite size effects impact on the phase diagrams, polar and dielectric properties of $(\mathrm{Sr}, \mathrm{Bi}) \mathrm{Ta}_{2} \mathrm{O}_{9}$ ferroelectric nanoparticles, J. Appl. Phys. 119 (2016) 204104.

[61] H. Sahwan, T. Choi, J.H. Jeon, Y. Kim, H. Lee, H.-Y. Joo, I. Hwang et al., Large resistive switching in ferroelectric $\mathrm{BiFeO}_{3}$ nano-island based switchable diodes, Adv. Mater. 25 (2013) 2339-2343.

[62] S. Takuya, K. Okada, A. N. Hattori, T. Kanki, A. S. Borowiak, B. Gautier, B. Vilquin, and H. Tanaka, Epitaxial inversion on ferromagnetic $(\mathrm{Fe}, \mathrm{Zn})_{3} \mathrm{O}_{4}$ /ferroelectric $\mathrm{BiFeO}_{3}$ core-shell nanodot arrays using three dimensional nanoseeding assembly, J. Appl. Phys. 113 (2013) 104302.

[63] Z. Xingang, B. Wang, X. Wang, X. Xiao, Z. Dai, W. Wu, J. Zheng, F. Ren, and C. Jiang, Preparation of BiFeO nanocomposites $(\mathrm{M}=\mathrm{Ag}, \mathrm{Au})$ bowl arrays with enhanced visible light photocatalytic activity, J. Am. Ceram. Soc. 98 (2015) 2255-2263.

[64] A. K. Tagantsev and G. Gerra, Interface-induced phenomena in polarization response of ferroelectric thin films, J. Appl. Phys. 100 (2006) 051607.

[65] M.D. Glinchuk, E.A. Eliseev, A.N. Morozovska, R Blinc, Giant magnetoelectric effect induced by intrinsic surface stress in ferroic nanorods, Phys. Rev. B 77 (2008) 024106.

[66] D. Rahmedov, S. Prosandeev, J. Íniguez, L. Bellaiche, Magnetoelectric signature in the magnetic properties of antiferromagnetic multiferroics: Atomistic simulations and phenomenology, Phys. Rev. B. 88 (2013) 224405.

[67] M.D. Glinchuk, E.A. Eliseev, Y. Gu, L-G Chen, V. Gopalan, A.N. Morozovska, Electric-field induced ferromagnetic phase in paraelectric antiferromagnets, Phys. Rev. B 89 (2014) 1014112.

[68] M.D. Glinchuk, E.A. Eliseev, A.N. Morozovska, New room temperature multiferroics on the base of single-phase nanostructured perovskites, J. Appl. Phys. 116 (2014) 054101.

[69] S. Prosandeev, I.A. Kornev, L. Bellaiche, Magnetoelectricity in $\mathrm{BiFeO}_{3}$ films: First-principles based computations and phenomenology, Phys. Rev. B 83 (2011) 020102.

[70] A.P. Pyatakov and A.K. Zvezdin, Flexomagnetoelectric interaction in multiferroics. Eur. Phys. J. B 71 (2009) 419.

[71] A. N. Morozovska, V.V. Khist, M.D. Glinchuk, V.Gopalan, and E.A. Eliseev, Linear antiferrodistortiveantiferromagnetic effect in multiferroics: Physical manifestations, Phys. Rev. B 92 (2015) 054421. 